\title{
Quantitative optimization of emergency department's nurses of an educational hospital: a case study
}

\author{
Mohammad Hosein Mehrolhasani ${ }^{1}$, Ali Mouseli ${ }^{2}$, Leila Vali ${ }^{3}$, Zahra Mastaneh ${ }^{4}$
}

${ }^{1}$ Associate Professor, Research Center for Health Services Management, Institute of Futures Studies in Health, Kerman University of Medical Sciences, Kerman, Iran

${ }^{2} \mathrm{Ph}$.D. Student in Health Services Management, Modeling in Health Research Center, Institute for Futures Studies in Health, Kerman University of Medical Sciences, Kerman, Iran

${ }^{3}$ Assistant Professor, Environmental Health Engineering Research Center, Kerman University of Medical Sciences, Kerman, Iran

${ }^{4}$ Assistant Professor, Social Determinants in Health Promotion Research Center, Hormozgan University of Medical Sciences, Bandar Abbas, Iran

\section{Type of article: Original}

\begin{abstract}
Introduction: Nurses account for the majority of human resources in hospitals, as such that $62 \%$ of the workforce and $36 \%$ of hospital expenditures are related to nurses. Considering its vital role in offering round-theclock emergency healthcare services, an Emergency Department (ED) requires adequate nurses. Therefore, this study was conducted to optimize the number of nurses in ED.

Methods: This was an applied study conducted using a Linear Programming (LP) model in 2015. The study population were selected by census who were all ED nurses $(n=84)$ and patients referred to ED $(n=3342)$. To obtain the statistics related to the number of patients and nurses, the hospital information system and human resources database were employed respectively. To determine the optimum number of nurses per shift, LP model was created via literature review and expert advice, and it was executed in WinQSB software.

Results: Before implementing the model, the number of nurses required for ED morning shift, evening shift, and night shift (2 shifts) was 26, 24 and 34 respectively. The optimum number of nurses who worked in ED after running the model was 62 nurses, 17 in the morning shift, 17 in the evening shift and 28 in the night shift (2 shifts). This reduced to 60 nurses after conducting sensitivity analysis.

Conclusion: The estimated number of nurses using LP was less than the number of nurses working in ED. This discrepancy can be reduced by scientific understanding of factors affecting allocation and distribution of nurses in ED and flexible organization, to reach the optimal point.
\end{abstract}

Keywords: Emergency Department; Nurses; Quantitative Optimization; Linear Programming Model

\section{Introduction}

Since the early $21^{\text {st }}$ century, the role of Human Resources (HR) in the health system has been recognized by international organizations such as the World Health Organization (1). Specialized and trained HR is the heart of any health system that ensures its life and survival (2). In this regard, providing specialized HR in hospitals as the most pragmatic unit of health system (3) is of special importance, since it accounts for 50 to $80 \%$ of expenditures in this sector (4). Nurses often account for the major part of HR in hospitals (5), such that Dehghan, cited by Eastaugh has regarded $62 \%$ of hospitals' HR and $36 \%$ of its expenditures related to nursing staff (6). This group plays a major role in promotion of health in society, without whom the hospitals will not achieve their goals (5). Of sectors, the success of which depends on having efficient and vigorous nurses along with other medical staff, is the Emergency Department (ED) which is an essential and inevitable element of hospitals considering its vital role in providing

\section{Corresponding author:}

Assistant Professor Dr. Zahra Mastaneh, Department of Health Information Management and Technology, Faculty of Para-Medicine, Hormozgan University of Medical Sciences, Bandar Abbas, Iran.

Tel: +987633666667 Email: zahramastaneh@yahoo.com

Received: April 12, 2016, Accepted: July 12, 2016, Published: February 2017

iThenticate screening: July 09, 2016, English editing: November 22, 2016, Quality control: December 10, 2016

(C) 2017 The Authors. This is an open access article under the terms of the Creative Commons Attribution-NonCommercialNoDerivs License, which permits use and distribution in any medium, provided the original work is properly cited, the use is non-commercial and no modifications or adaptations are made. 
immediate medical care for patients who require quick intervention at all times of the day (7). Due to the critical condition of patients referring to ED and hospitalization of over $28 \%$ of them in various wards of a hospital, the quality of services provided in this department is regarded as a symbol of the general state of services provided in the hospital (8). Providing suitable and programmed HR, namely nursing staff for ED is definitely one of the major tasks of hospital administrators who should employ orderly and rational processes and systemic approaches to avoid shortage or surplus (9); since, on the one hand, hospitals encounter resource constraints and rising costs, and on the other hand, they should be accountable to medical requirements of patients and satisfy their expectations and requirements (6). In this regard, since 1980, many authors have investigated scheduling of various medical staff, including ED nurses, as such that the common objectives of all these studies are to determine the least number of nurses required along with patients' satisfaction of emergency services provided, as well as to determine the number of nurses required per shift (10). Within recent decades, various tools including statistics, work measurement, simulation, queues and linear programming models have been used for scheduling problems of staff (11) as such that within the last two decades, the use of simulation models for programming and decision-making in the health sector has been extensively promoted and many of them have been used in the ED of hospitals (12). In 2009, Ahmed and Alkhamis provided a tool to support decision-making using a simulation system along with optimization, to determine the optimal number of physicians, laboratory technicians and nurses for a hospital in Kuwait, in order to maximize the number of patients and reduce waiting time in ED, considering budget constraints. The main objective of this tool was to evaluate the effect of optimal allocation of staff on efficiency of provided services. Results showed that the use of optimization model results in a $28 \%$ increase in customers (patients) and a $40 \%$ decrease in patients' waiting time (13). In this regard, various studies have demonstrated the appropriateness of a Linear Programming (LP) model in optimal allocation of health system resources relative to other traditional methods. For instance, Flessa (2000) and Beaulieu et al. (2000) employed this method respectively for optimal allocation of resources in developing countries (14) and to schedule shifts for physicians and nurses in ED. Results from employing this method showed a significant decrease in time and intended activity, and better scheduling in a 6month interval (15). This widely used model is a set of techniques and methods derived from mathematics and other disciplines that is considerably effective in improving administrative decisions and finding optimal conditions and it can suitably solve problems like production planning, allocation of limited resources and inquiry control. Managers who are interested in the quality of their decisions' results, cannot be indifferent, relative to this issue (16). Relative to other models including complex ones, this model is of more use due to being simple and feasible with data available in EDs (11). The ED of the educational hospital under study in Bandar Abbas, as the largest ED in the south of Iran, admits a high number of patients from all counties of the province and even adjacent provinces for emergency services. Therefore, timely provision of these services, proportional to patients' needs, requires an adequate number of trained nurses. In addition, since the LP model is one of the widely used optimization methods for HR allocation proportional to the demand and workload, this study was conducted with the aim of estimating the optimum number of nurses using the LP model in the ED of the intended hospital.

\section{Material and Methods}

This was an applied study, conducted using the quantitative technique of Linear Programming (LP). LP entails 3 main components of decision variables, objective function and constraints or limitations, and appropriate definition of decision variables is the first major step to extend the model. Then objective function and constraints are determined. Decision variables are those that values should be attributed to. Objective function shows the relations between variables and objective of the problem and it seeks for optimization. Limitations are those constraints that should be observed (17). This study was conducted using available data in 2015 when there was the high admission rate of patients. Statistical population was selected taking a census including all patients $(3,342$ patients) referring to the ED of the educational hospital in Bandar Abbas in the above-mentioned period as well as nurses working in this ward (84 nurses). Data collection was conducted using the Hospital Information System (HIS) for the number of patients, and the hospital HR database for data related to nurses. To verify the number of nurses working at ED being up-to-date at the time of this study, data were reviewed by the nursing manager of the hospital. Furthermore, confidentiality of obtained data was confirmed and they were used only for research purposes. Work shifts of the hospital under study were designed as two 6-hour shifts ( 8 am to $2 \mathrm{pm}$ and $2 \mathrm{pm}$ to $8 \mathrm{pm}$ ) and a 12 -hour shift (8 pm to $8 \mathrm{am}$ ). To determine the mean admission of patients with sufficient validity and planning of the LP model, a $24-$ hour period of this ward was divided into eight periods of 3-hours according to Table 1 . According to the study by Ketabi and Monzavi (11), patients referring to ED require one of low, medium and high levels of care and duration of nursing services for each one of these levels is respectively 10, 20 and 45 minutes. On average, in each period, $40 \%$ of patients require low level nursing services, $20 \%$ need medium level and 40\% need high level nursing services. To determine the optimum number of nurses for ED per various shifts of the hospital, data entered Excel 
software and descriptive statistics indices were extracted. To design the LP model, the initial model was extracted using literature review and it was finalized by specialized counseling. Then, using collected field data, the model was executed in WinQSB software. This software is among the simplest and yet the most accurate of engineering software able to solve various problems in a simple environment as a software package, by easy data entry, various solving methods, analyzing all parameters and understandable output (11).

Table 1. Current eight shifts of the ED

\begin{tabular}{|l|l|l|l|}
\hline \multicolumn{2}{|l|}{ Current shifts of the ED } & Division of eight shifts in ED \\
\hline Shifts & Period & Shifts & Period \\
\hline First & $8 \mathrm{am}-2 \mathrm{pm}$ & First & $8 \mathrm{am}-11 \mathrm{am}$ \\
\cline { 3 - 4 } & & Second & $11 \mathrm{am}-2 \mathrm{pm}$ \\
\hline Second & $2 \mathrm{pm}-8 \mathrm{pm}$ & Third & $2 \mathrm{pm}-5 \mathrm{pm}$ \\
\cline { 3 - 4 } & & Fourth & $5 \mathrm{pm}-8 \mathrm{pm}$ \\
\hline \multirow{2}{*}{ Third } & \multirow{2}{*}{$8 \mathrm{pm}-8 \mathrm{am}$} & Fifth & $8 \mathrm{pm}-11 \mathrm{pm}$ \\
\cline { 3 - 4 } & & Sixth & $11 \mathrm{pm}-2 \mathrm{am}$ \\
\cline { 3 - 4 } & & Seventh & $2 \mathrm{am}-5 \mathrm{am}$ \\
\hline & & Eighth & $5 \mathrm{am}-8 \mathrm{am}$ \\
\hline
\end{tabular}

\section{Results}

The number of patients referring to ED within the study period was 3,342, and average admission of patients was 1,114. Considering the average number of customers in 3 -hour shifts as well as the time required to supply nursing services for each group of patients (low, medium and high level care), and the percentage of patients who received low, medium and high level care, the number of nurses required to supply services for customers was obtained for each hour in Table 2.

Table 2. Time served, the number of patients, and nurses required at any shifts of the ED

\begin{tabular}{|l|l|l|l|l|l|}
\hline Shifts & Period & $\begin{array}{l}\text { Total number of patients } \\
\text { referred in studied period }\end{array}$ & $\begin{array}{l}\text { Average number of } \\
\text { patients per shift }\end{array}$ & $\begin{array}{l}\text { Nursing services } \\
\text { required (min) }\end{array}$ & $\begin{array}{l}\text { Number of nurses } \\
\text { needed per shift }\end{array}$ \\
\hline First & $8 \mathrm{am}-11 \mathrm{am}$ & 360 & 40 & 1040 & 17 \\
\hline Second & $11 \mathrm{am}-2 \mathrm{pm}$ & 732 & 81 & 2106 & 35 \\
\hline Third & $2 \mathrm{pm}-5 \mathrm{pm}$ & 360 & 40 & 1040 & 17 \\
\hline Fourth & $5 \mathrm{pm}-8 \mathrm{pm}$ & 480 & 53 & 1378 & 23 \\
\hline Fifth & $8 \mathrm{pm}-11 \mathrm{pm}$ & 300 & 33 & 858 & 14 \\
\hline Sixth & $11 \mathrm{pm}-2 \mathrm{am}$ & 135 & 15 & 390 & 6 \\
\hline Seventh & $2 \mathrm{am}-5 \mathrm{am}$ & 150 & 17 & 442 & 7 \\
\hline Eighth & $5 \mathrm{am}-8 \mathrm{am}$ & 825 & 92 & 2392 & 40 \\
\hline
\end{tabular}

The above-mentioned cases were used as the input for the LP model. The objective function of this model was the minimum number of nurses required for the ED of the hospital. In addition, the objective function, decision variables and constraints are as follows:

a) Objective function:

Min $\mathrm{Z}=\mathrm{X} 1+\mathrm{X} 2+\mathrm{X} 3+\mathrm{X} 4+\mathrm{X} 5+\mathrm{X} 6+\mathrm{X} 7+\mathrm{X} 8$

To determine decision variables, it is necessary to note that according to current shift schedule of the hospital as well as the times of this model, each shift can have nurses of various times, such that the morning shift ( $8 \mathrm{am}-2 \mathrm{pm})$ could have nurses from the first period $(8 \mathrm{am}-11 \mathrm{am})$ and the second period $(11 \mathrm{am}-2 \mathrm{pm})$. In evening shift $(2 \mathrm{pm}-8$ $\mathrm{pm})$, it was possible to have nurses from the third $(2 \mathrm{pm}-5 \mathrm{pm})$ and fourth $(5 \mathrm{pm}-8 \mathrm{pm})$ periods. Furthermore, the night shift $(8 \mathrm{pm}-8 \mathrm{am})$ could entail nurses from fifth $(8 \mathrm{pm}-11 \mathrm{pm})$, sixth $(11 \mathrm{pm}-2 \mathrm{am})$, seventh $(2 \mathrm{am}-5 \mathrm{am})$ and eighth (5 am-8 am) periods.

b) Decision variables of above model were as follows:

$\mathrm{X} 1$ : The number of nurses whose shift started at $8 \mathrm{am}$.

$\mathrm{X} 2$ : The number of nurses whose shift started at 11 am (null).

$\mathrm{X} 3$ : The number of nurses whose shift started at $2 \mathrm{pm}$.

$\mathrm{X} 4$ : The number of nurses whose shift started at $5 \mathrm{pm}$ (null).

X5: The number of nurses whose shift started at $8 \mathrm{pm}$.

X6: The number of nurses whose shift started at $11 \mathrm{pm}$ (null). 
X7: The number of nurses whose shift started at 2 am (null).

X8: The number of nurses whose shift started at 5 am (null).

c) Constraints:

c.1. Time-constraints (demand): According to these constraints, the number of nurses required for each period was as follows:

$\mathrm{X} 1 \geq 17$

$\mathrm{X} 1+\mathrm{X} 2 \geq 35$

$\mathrm{X} 3 \geq 17$

$\mathrm{X} 3+\mathrm{X} 4 \geq 23$

$\mathrm{X} 5 \geq 14$

$X 5+X 6 \geq 6$

$\mathrm{X} 5+\mathrm{X} 6+\mathrm{X} 7 \geq 7$

$\mathrm{X} 5+\mathrm{X} 6+\mathrm{X} 7+\mathrm{X} 8 \geq 40$

c.2. Supply constraints: This hospital encountered no constraints for supplying nurses in various work shifts.

c.3. Constraints of non-negative variables:

$\mathrm{X} 1, \mathrm{X} 2, \mathrm{X} 3, \mathrm{X} 4, \mathrm{X} 5, \mathrm{X} 6, \mathrm{X} 7, \mathrm{X} 8 \geq 0$

After solving the model in WinQSB software, the optimum number of nurses required for ED in three work shifts of the hospital was 48 nurses (Table 3 ) and considering that the third shift is a 12-hour shift, the actual number of nurses required for a 24-hour cycle was $(48+14)=62$ nurses.

Table 3. The number of nurses required per shift in the ED of the hospital before and after running the model

\begin{tabular}{|l|l|l|}
\hline Shifts & $\begin{array}{l}\text { Number of nurses needed before } \\
\text { running the model }\end{array}$ & $\begin{array}{l}\text { Number of nurses needed after } \\
\text { running the model }\end{array}$ \\
\hline First $(8 \mathrm{am}-2 \mathrm{pm})$ & 26 & 17 \\
\hline Second $(2 \mathrm{pm}-8 \mathrm{pm})$ & 24 & 17 \\
\hline Third & $17+17$ & $14+14$ \\
\hline $\begin{array}{l}\text { Total number of nurses } \\
\text { needed per day }\end{array}$ & 84 & 62 \\
\hline
\end{tabular}

If the current shifts of the hospitals are changeable and nurses are allowed to start their work in various shifts, a lower number of nurses will be required due to fewer constraints. In other words, in the solved model, since the third shift starts at $8 \mathrm{pm}$ and continues to $8 \mathrm{am}, 14$ nurses whose presence is required from $8 \mathrm{pm}$ to $11 \mathrm{pm}$ are practically at work until 8 am and the cost of their surplus is imposed to the hospital. However, if nurses can start work from the middle of current shifts, in the case of re-solving the LP model, the number of nurses required for every shift and the objective function will change as follows:

a) Objective function

$\mathrm{Min} \mathrm{Z}=\mathrm{X} 1+\mathrm{X} 2+\mathrm{X} 3+\mathrm{X} 4+\mathrm{X} 5+\mathrm{X} 6+\mathrm{X} 7+\mathrm{X} 8$

b) Decision variables

$\mathrm{X} 1$ : The number of nurses whose shift started at $5 \mathrm{am}$.

$\mathrm{X} 2$ : The number of nurses whose shift started at $8 \mathrm{am}$.

$\mathrm{X} 3$ : The number of nurses whose shift started at $11 \mathrm{am}$.

$\mathrm{X} 4$ : The number of nurses whose shift started at $2 \mathrm{pm}$.

$\mathrm{X} 5$ : The number of nurses whose shift started at $5 \mathrm{pm}$.

X6: The number of nurses whose shift started at $8 \mathrm{pm}$.

X7: The number of nurses whose shift started at $11 \mathrm{pm}$.

X8: The number of nurses whose shift started at 2 am.

c) Constraints:

c.1. Constraints of time (demand):

Based on these constraints, the number of nurses required in each time was as follows, based on new work shifts:

$\mathrm{X} 8+\mathrm{X} 1 \geq 17$

$\mathrm{X} 1+\mathrm{X} 2 \geq 17$

$\mathrm{X} 2+\mathrm{X} 3 \geq 35$

$\mathrm{X} 3+\mathrm{X} 4 \geq 17$

$\mathrm{X} 4+\mathrm{X} 5 \geq 23$

$\mathrm{X} 5+\mathrm{X} 6 \geq 14$ 
$\mathrm{X} 6+\mathrm{X} 7 \geq 6$

$\mathrm{X} 7+\mathrm{X} 8 \geq 7$

c.2. Supply constraints: None of the nurses were willing to start working at $2 \mathrm{am}, \mathrm{X} 8=0$.

c.3. Constraints of non-negative variables:

$\mathrm{X} 1, \mathrm{X} 2, \mathrm{X} 3, \mathrm{X} 4, \mathrm{X} 5, \mathrm{X} 6, \mathrm{X} 7, \mathrm{X} 8 \geq 0$

$\mathrm{X} 1=17, \mathrm{X} 2=0, \mathrm{X} 3=17, \mathrm{X} 4=0, \mathrm{X} 5=14, \mathrm{X} 6=6, \mathrm{X} 7=6$

In addition, the least number of nurses required at this state was $z=60$ that compared to previous state, 2 fewer nurses were required.

\section{Discussion}

Determining the optimum number of human resources in EDs of hospitals has been investigated in several studies $(11,18-20)$. Based on results of these studies, considering the importance of this ward and the emergency nature of its functions, absence of an optimum number of human resources can directly affect the quality of activities performed in this ward as well as patients' satisfaction. Low number of human resources, namely nurses, can disrupt the quality and quantity of services provided in this ward, and result in crowding of patients and lack of receiving timely care. On the other hand, an excess number of nurses will result in imposition of excess expenses to the hospital and in addition, will make organization and coordination of them more difficult $(19,20)$. Therefore, determining the optimum number of nurses is the main task of managers in this ward, and this study confirmed that its importance was similar to other studies. In this study, using an LP model, the optimum number of nurses was estimated to be 62. This value in Ketabi's study was 36 nurses, in Sotodezadeh it was seven and in Bayati's study it was three $(11,18,21)$. The estimated number of nurses in these studies is different depending on the type and size of the hospital, as well as the number of referring patients. In this study, this number is higher compared to similar studies, since the hospital under study is the largest hospital in the province and many patients from all over the province refer or are referred to the ED of this hospital. Considering the number of nurses working in this ward ( 84 nurses), 22 are determined as extra forces. This number is higher than the optimum value and it clearly imposes extra expenses to the hospital. Centeno (2003) who had employed simulation and LP in combination to determine the optimum number of ED nurses, showed that the number of nurses estimated, using these methods, is lower compared to the number of nurses calculated experimentally (10), which complies with the results of present study. Current hospital shifts impose many constraints in determining the optimum number of nurses. In this regard, by changing the number of shifts, the start point of each shift and its duration, employing sensitivity analysis in various studies including the present study, has sought to reduce these constraints to employ a variable number of nurses in shorter shifts and this will reduce the total number of nurses. In sensitivity analysis, the estimated number of patients was lower compared to the estimated number resulted from the LP (11). This reduction in present study was two nurses and Ketabi's study shows a reduction in required workforce of up to eight nurses (11). Therefore, flexibility in organizing work shifts and creating a culture of acceptance among nurses working at ED can assist allocating the optimum number of nurses that, while they are quantitatively adequate for each shift, no damage is imposed to the quantity and quality of services provided. Like similar studies, results of this study showed that the LP model is employed as a practical model to determine the optimum number of nurses in many cases. In this regard, Ketabi stated that this model can not only determine the optimum number of nurses, but can also determine the number of nurses required for each work shift (11). However, this model suffers from some limitations including being singleobjective and an inflexibility that disables groups' decision-making (22). Therefore, to solve optimization problems, including the optimum number of HR, it is suggested that an ideal programming model is used with the capability of selecting multi-objectives, flexibility in decision-making and considering preferences of various decision-making groups for multiple and sometimes contradictory objectives. Considering the lack of access to accurate data of all months of the year and selecting only some months, as well as lack of access to complete data to estimate the time required to provide nursing services, and using data from similar studies, this study encountered some limitations. To remove them, it was attempted to use data of the months with the highest number of customers based on which the highest number of nurses was estimated. In addition, data from a relatively similar study was employed, to determine the time of providing nursing services as well as modeling assumptions. Finally, it is suggested that accurate and complete data be provided, to create model assumptions according to the demands of the hospital and to employ flexible models to determine the optimum number of nurses.

\section{Conclusions}

In summary, findings of this study showed that the estimated number of patients using the LP model was much lower than nurses working at the ED of the hospital under study. The functional significance of these findings is that by using scientific understanding of factors affecting allocation and distribution of nurses in ED and their flexible 
organization, the number of nurses can reach the optimum value. Therefore, it is suggested to conduct shift scheduling using sensitivity analysis such that it is proportional to the demand of services in various hours of a day to satisfy demands and reduce the burden of HR expenses. Finally, conducting a complementary study for quantitative optimization of nurses in all clinical wards of the hospital can be a suitable course for future studies on this issue.

\section{Acknowledgments:}

This study is the result of a project approved by the Research Center of Health Services Management at Kerman University of Medical Sciences, N. 93/151 and the authors appreciate officials of this center as well as the nursing manager of the hospital and the nurses working at the ED of the educational hospital in Bandar Abbas.

\section{Conflict of Interest:}

There is no conflict of interest to be declared.

Authors' contributions:

All authors contributed to this project and article equally. All authors read and approved the final manuscript.

\section{References:}

1) World Health Organization. The world health report 2006-working together for health. World Health Organization. 2006. Available from: http://www.who.int/whr/2006/en/

2) Global Health Trust. Human resources for health and development: A joint learning initiative. Global Health Trust. 2003.

3) Mastaneh Z, Mouseli L, Jahangiri M, Doust M, Eshghi A. Strength and weakness of crisis management in Hormozgan Medical University's Hospitals. JFUMS. 2012; 1(4): 244-50.

4) Mastaneh Z, Mouseli L, Zamani M, Boromand E, Dadipoor S, Beizaei F, et al. Assessment of hospitalization pattern of training hospitals in Bandar-abbas based on the International Classification of Diseases during 2002-2007. IJS. 2011; 19(1): 1-11.

5) Mastaneh Z, Mouseli L, Zamani M, Boromand F, Dadipoor S, Beizaei F, et al. Investigation of nursing job satisfaction in university hospitals affiliated to Hormozgan University of Medical Sciences. HMJ. 2014; 18(3): 283-9.

6) Dehghan Nayeri N, Nazari AA, Salsali M, Ahmadi F. To assess role of staffing in nursing productivity: a qualitative research. HAYAT. 2006: 12(3): 5-15.

7) Jabbari A, Jafarian M, Khorasani E, Ghaffari M, Majlesi M. Emergency Department waiting time at Alzahra hospital. JIM. 2011; 8(4(20)): 500-11.

8) Heydaranlou E, Khaghani Zadeh M, Ebadi A, Sirati Nir M, Aghdasi Mehr, Abad N. A survey on implementation of FOCUS-PDCA on performance of Tabriz Shahid Mahalati emergency department. JAUMS. 2008; 10(4): 5-9.

9) Swansburg RC, Swansburg RJ. Introductory management and leadership for nurses. Boston: Jones and Bartlett Publisher. 2000.

10) Centeno M, Giachetti R, Linn R, Ismail A. A Simulation -ILP based tool for scheduling ER staff. Proceedings of the 2003 Winter Simulation Conference, Morrice. 2003; 1930-8. doi: 10.1109/WSC.2003.1261656.

11) Ketabi S, Monzavibarzaki J. Quantitative optimization of emergency department's nursing staff at Chamran hospital using linear programming method. JIM. 2006; 3(2): 83-91.

12) Noori Hekmat S, Dehnavieh R, Mohamadi N, Poorhosseini S, Rezaei F, Mehrolhasani M, et al. Investigation of optimal number of nursing in emergency department of Shafa center, Kerman. TBJ. 2014; 13(2): 140-55.

13) Ahmed MA, Alkhamis TM. Simulation optimization for an emergency department healthcare unit in Kuwait. EJOR. 2009; 198(3): 936-42. doi: 10.1016/j.ejor.2008.10.025.

14) Flessa S. Where efficiency saves lives: A linear programme for the optimal allocation of health care resources in developing countries. Health Care Manag Sci. 2000; 3(3): 249-67. PMID: 10907327.

15) Beaulieu H, Ferland JA, Gendron B, Michelon P. A mathematical programming approach for scheduling physicians in the emergency room. HCMS. 2000; 3(3): 193-200. doi: 10.1023/A:1019009928005.

16) Mehdipor I, Sadrolashrafi SM, Karbasi AR. Meaty chicken' feed framing with linear programming model. JAS. 2006; 12(3): 479-87. 
17) Hamdi AT. Introduction to operations research. Translated by: Mehdi Toloe and Mohammad Reza Alirezaie. 7th Ed. Tehran: Center for Academic Publication. 2010.

18) Bayati M, Kharazmi E, Javanbakht M, Sadeghi A, Arefnejad M, Vahedi S, et al. Optimization the number of nurses in the emergency department using linear programming technique. JHMI. 2014; 1(2): 41-5.

19) Wang X. Emergency department staffing: A separated continuous linear programming approach. Math Probl Eng. 2013; 1-8. doi: 10.1155/2013/680152.

20) Al-Najjar SM, Ali SH. Staffing and scheduling emergency rooms in two public hospitals: A Case Study. IJBA. 2011; 2(2): 137-48. doi: 10.5430/ijba.v2n2p137.

21) Sotodezade F, Kavosi Z, Bayati M, Azami S. Optimization of nursing number in poisoning department of Shoshtari hospital of Shiraz using linear programming. First National Continuous Quality Improvement and Clinical Governance Conference. Tabriz University of Medical Sciences. 2011.

22) Momeni M. New topics in operation research. 5th Ed. Tehran. Moalleph. 2013. 